\title{
Analytical growth equations and their Genstat 5 equivalents
}

\section{HEINEN}

DLO Research Institute for Agrobiology and Soil Fertility (AB-DLO), PO Box 14, NL-6700 AA Wageningen, The Netherlands

Fax: +31-317-423110; e-mail: m.heinen@ab.dlo.nl

Received 2 July 1998; Accepted 14 January 1999

\begin{abstract}
This paper compares two ways of representing some of the existing growth functions, i.e., the exponential, the monomolecular or Mitscherlich, the logistic or autocatalytic, the Gompertz, and the Richards equations. A second order exponential polynomial will be discussed as well. In the first expression growth is expressed in the parameters mass at time zero $W_{0}$, mass at time infinity $W_{f}$, and a measure for the relative growth rate $k$. In the second expression different parameters are used because of robust parameter optimization (e.g., by the statistical software package Genstat). This paper shows the relationships between these fitted parameters and the parameters $W_{0 s} W_{f}$ and $k$. This paper addresses the properties of these models, such as physical meaning of the parameters, properties at the point of inflection (if existing), and the growth rate in the limit $W \rightarrow 0$. The second order exponential polynomial is rewritten in such way that use is made of a proportionality constant, being equal to the relative growth rate at point of inflection. Application of the growth models is demonstrated using data of lettuce grown on nutrient film. Finally, it is shown that, except for the exponential polynomial, all growth equations originate from one single equation.
\end{abstract}

Keywords: growth rate, relative growth rate, analytical growth equation, exponential growth, monomolecular or Mitscherlich growth, logistic or autocatalytic growth, Gompertz growth, Richards or general logistic growth, exponential polynomial growth, point of inflection

\section{Introduction}

Growth of a crop involves uptake of water and nutrients. Because nutrients are taken up by the plant to be incorporated in new plant material, study of the increase in plant mass gives a first idea of the need of nutrients by the plant. In case a nutrient constitutes a constant fraction of the plant mass, the uptake rate is proportional to the growth rate of the plant.

Descriptions of growth rate, i.e. increase in plant mass with time, can be given either by physiological mechanistic models or by empirical or mathematical functions. Mechanistic models are mostly used to predict growth, while mathematical functions are mostly used to describe afterwards observed increase in mass. By fitting the observed data to the relatively simple mathematical functions, the parameters appear- 


\section{HEINEN}

ing in these functions are optimized. It appears that there are several ways to represent the same mathematical function. That means that the parameters appearing in each representation of the same function are linked to each other. This paper describes a few mathematical growth functions that appear in the literature. These functions are formulated using parameters with a physiological meaning that can be identified as mass at time zero, mass at time infinity, and a proportionality constant that is a measure for the relative growth rate. These functions appear also in a specific statistical package (i.c. Genstat 5; Anon., 1993), but then they are formulated in a different way, for reasons of robust parameter optimization. The aim of this paper is to show how the parameters appearing in the two descriptions of the same mathematical function are related, and to show some of the properties of these functions. As a demonstration, examples of fitting growth and nutrient uptake data to some of these functions are presented. Finally it is shown that most of the growth functions used in this paper belong to one family of equations. This paper is not meant to give a review of existing growth functions; such reviews can be found in existing handbooks (e.g., Charles-Edwards et al., 1986; France and Thornley, 1984; Hunt, 1978, 1982).

\section{Mathematical growth models}

All growth models considered in this study explicitly express the plant mass as a function of time. They are, however, empirical models, i.e., growth is not explained in terms of physiological processes. Only later the parameters of these growth equations can be given a physiological meaning. For this study use is made of standard references (Charles-Edwards et al., 1986; France and Thornley, 1984; Hunt, 1978, 1982).

In general, the growth rate will be the starting point, i.e. the change in plant mass $W(\mathrm{M})$ with time $t(\mathrm{~T}) \mathrm{d} W / \mathrm{d} t$, or the growth rate per unit plant mass $(1 / W)(\mathrm{d} W / \mathrm{d} t)$, known as the relative or specific growth rate or sometimes proportional growth rate. Here growth is considered to be a function of the plant mass already formed:

$$
\frac{\mathrm{d} W}{\mathrm{~d} t}=f(W)
$$

Now the question remains how this function $f(W)$, which relates $\mathrm{d} W / \mathrm{d} t$ and $W$, looks like. Several functions $f(W)$ will be considered, typically those that are present in Genstat 5 (Anon., 1993). For each of the functions the following expressions and properties will be determined.

A The main part of the model is the expression for $W(t)$, which is obtained by integrating the expression for $\mathrm{d} W / \mathrm{d} t$ between the limits $t=0, W=W_{0}$ and $t=t, W=$ $W$. The main parameters appearing in these equations are given a physiological meaning, such as the weight $W_{0}$ at $t=0$, the weight $W_{f}$ at $t \rightarrow \infty$, and the growth rate proportionality constant $k$, which is a measure for the relative growth rate.

B Some of the models have an inflection point. The plant mass $W^{*}$ and time $t^{*}$ at the inflection point are obtained by solving $\mathrm{d}^{2} W / \mathrm{d} t^{2}=0$ for $W$ and $t$, respectively. 

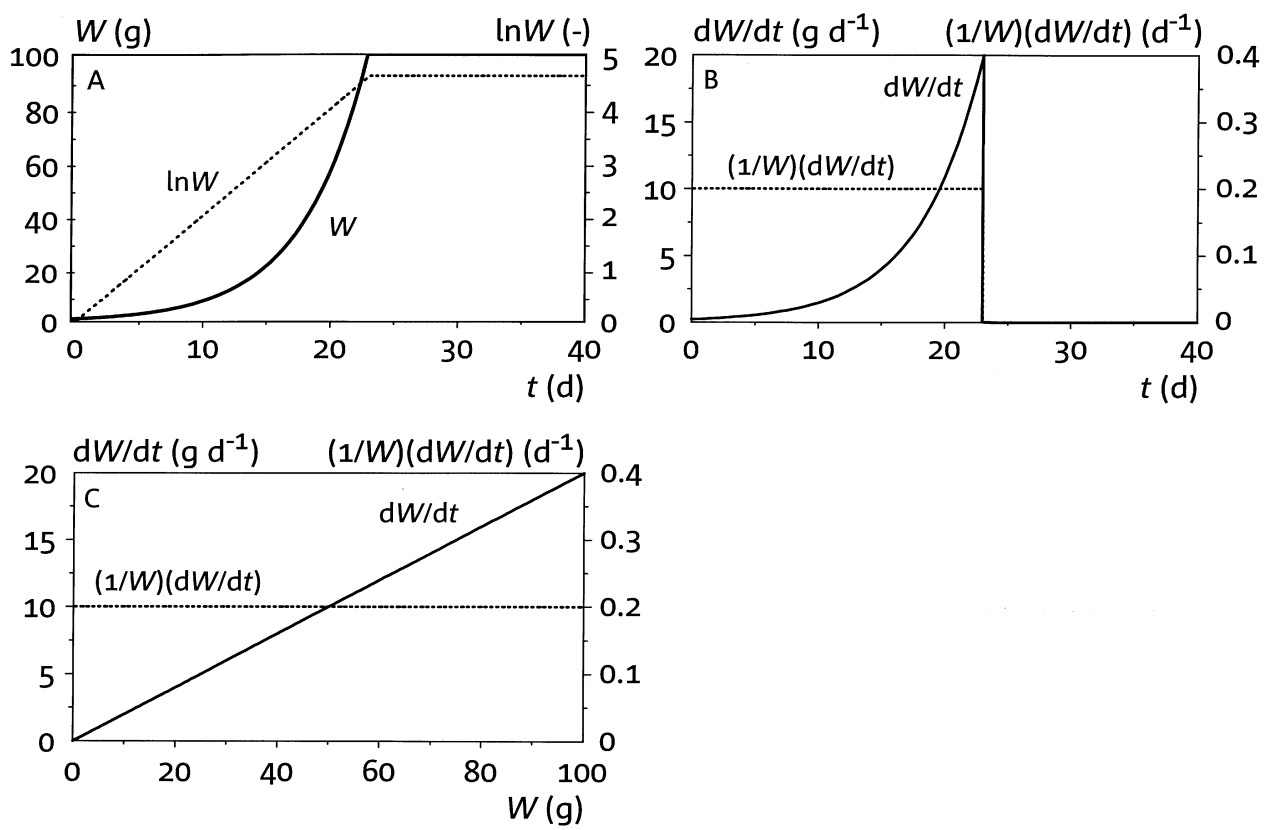

Figure 1. Exponential growth: (A) $W_{e}$ and $\ln W_{e}$ as a function of $t,(\mathrm{~B}) \mathrm{d} W_{e} / \mathrm{d} t$ and $\left(1 / W_{e}\right)\left(\mathrm{d} W_{e} / \mathrm{d} t\right)$ as a function of $t$, and (C) as function of $W_{e}$; with $W_{e 0}=1 \mathrm{~g}, W_{e f}=100 \mathrm{~g}, k_{e}=0.2 \mathrm{~d}^{-1}$.

Substituting $W^{*}$ into the expressions for growth rate and relative growth rate yields values for these rates at the inflection point, $(\mathrm{d} W / \mathrm{d} t)^{*}$ and $((1 / W)(\mathrm{d} W / \mathrm{d} t))^{*}$, respectively.

C One expects the growth rate for $W \rightarrow 0$ to be finite. To check this, the limit for $W \rightarrow 0$ of $\mathrm{d} W / \mathrm{d} t$ is determined, and for comparison also the limit for $W \rightarrow 0$ of $(1 / W)(\mathrm{d} W / \mathrm{d} t)$ is given.

The aspects of $\mathrm{A}$ are described in the forthcoming sections. The six models considered are: exponential, monomolecular or Mitscherlich, logistic or autocatalytic, Gompertz, Richards or general logistic, and second order exponential polynomial growth. The common parameters appearing in all models will be distinguished by the subscripts $e$, $m, l, g, r$, and $p$, respectively. For convenience, all main symbols with their dimensions are described in Table 1 and not in the text, and the expressions for $W(t)$ are summarized in Table 2. The properties mentioned in $\mathrm{B}$ and $\mathrm{C}$ are listed in Table 3, and will not be discussed in the text. Finally, for all models graphical representations are given of $W, \ln W, \mathrm{~d} W / \mathrm{d} t$ and $(1 / W)(\mathrm{d} W / \mathrm{d} t)$ as a function of $t$, and of $\mathrm{d} W / \mathrm{d} t$ and $(1 / W)(\mathrm{d} W / \mathrm{d} t)$ as a function of $W$. In all models the lower asymptote is absent.

\section{Exponential growth}

The growth rate is proportional to the amount of plant mass already formed. Growth is irreversible and stops at a certain time, $t_{f}(\mathrm{~T})$, e.g. when one of the growth factors 


\section{HEINEN}

Table 1. Description of symbols used in text including their dimensions (M: mass, T: time, 1: dimensionless).

\begin{tabular}{|c|c|c|}
\hline Symbol & Description & Dimension \\
\hline$A$ & Dummy parameter (Eq. (63), and Appendix A) & 1 \\
\hline$B$ & Dummy parameter (Appendix A) & $\mathbf{M}^{n-1}$ \\
\hline$W$ & Plant mass & M \\
\hline$Y$ & Plant mass & M \\
\hline$a, b, c, d$ & Constants in exponential polynomial (non-subscripted) & $1, \mathrm{~T}^{-1}, \mathrm{~T}^{-2}, \mathrm{~T}^{-3}$ \\
\hline$a, b, c$ & $\begin{array}{l}\text { Parameters in section 'Equivalent expressions for growth models' } \\
\text { (subscripted); } a \text { represents the lower asymptote, }(a+c) \text { represents the } \\
\text { upper asymptote, and } b \text { is a measure for the slope }\end{array}$ & $\mathrm{M}, \mathrm{T}^{-1}, \mathrm{M}$ \\
\hline$k$ & Proportionality constant & $\mathrm{T}^{-1}$ \\
\hline$k_{l}^{\prime}$ & Proportionality constant (for logistic growth only) & $\mathrm{M}^{-1} \mathrm{~T}^{-1}$ \\
\hline$k_{g}$ & Non-constant proportionality constant (for Gompertz growth only) & $\mathrm{T}^{-1}$ \\
\hline$m$ & Time of inflection & $\mathrm{T}$ \\
\hline$n$ & Dimensionless constant & 1 \\
\hline$p$ & Dimensionless constant & 1 \\
\hline$r$ & Dimensionless parameter & 1 \\
\hline$t$ & Time & $\mathrm{T}$ \\
\hline$t_{f}$ & Time when growth stops (for exponential growth only) & $\mathrm{T}$ \\
\hline$t_{m i}$ & Last time of measurement interval & $\mathrm{T}$ \\
\hline$t_{s}$ & The second order exponential polynomial is symmetrical at time $t=t_{s}$ & $\mathrm{~T}$ \\
\hline$y$ & Expected value of $Y$ & M \\
\hline$\alpha, \beta, \gamma$ & $\begin{array}{l}\text { Parameters in universal growth equation (Eq. (61)); dimensions depend on } \\
\text { choice of growth function (see, e.g., Table 6) }\end{array}$ & \\
\hline$\varepsilon$ & Constant (used in ln-transformation of exponential polynomial growth) & 1 \\
\hline$\mu, v$ & Curve shape parameters & $1, \mathrm{~T}^{-1}$ \\
\hline$\sigma$ & A stochastic deviation & M \\
\hline
\end{tabular}

Sub- and superscripts

$\begin{array}{ll}* & \text { Property at inflection point } \\ 0 & \text { Property at } t=0 \\ 1 & \text { Referring to some time at start of growth period } \\ 2 & \text { Referring to some time at end of growth period } \\ e & \text { Reference to the exponential growth function } \\ f & \text { Property for } t \rightarrow \infty \text {; for exponential growth: } t=t_{f} \\ g & \text { Reference to the Gompertz growth function } \\ l & \text { Reference to the logistic or autocatalytic growth function } \\ m & \text { Reference to the monomolecular or Mitscherlich growth function (not in } t_{m} \text { ) } \\ p & \text { Reference to the exponential polynomial growth function } \\ r & \text { Reference to the Richards growth function } \\ t & \text { ln-transformed data (exponential polynomial growth) }\end{array}$

Mathematical operators

d Derivative operator

e, exp Natural base

lim Limit operator

$f() \quad$ Any functional relationship

ln Natural logarithm

$\int \quad$ Integral operator 
Table 2. Names, expressions for $W(t)$, and reference to equation number in text for the growth equation used in this paper.

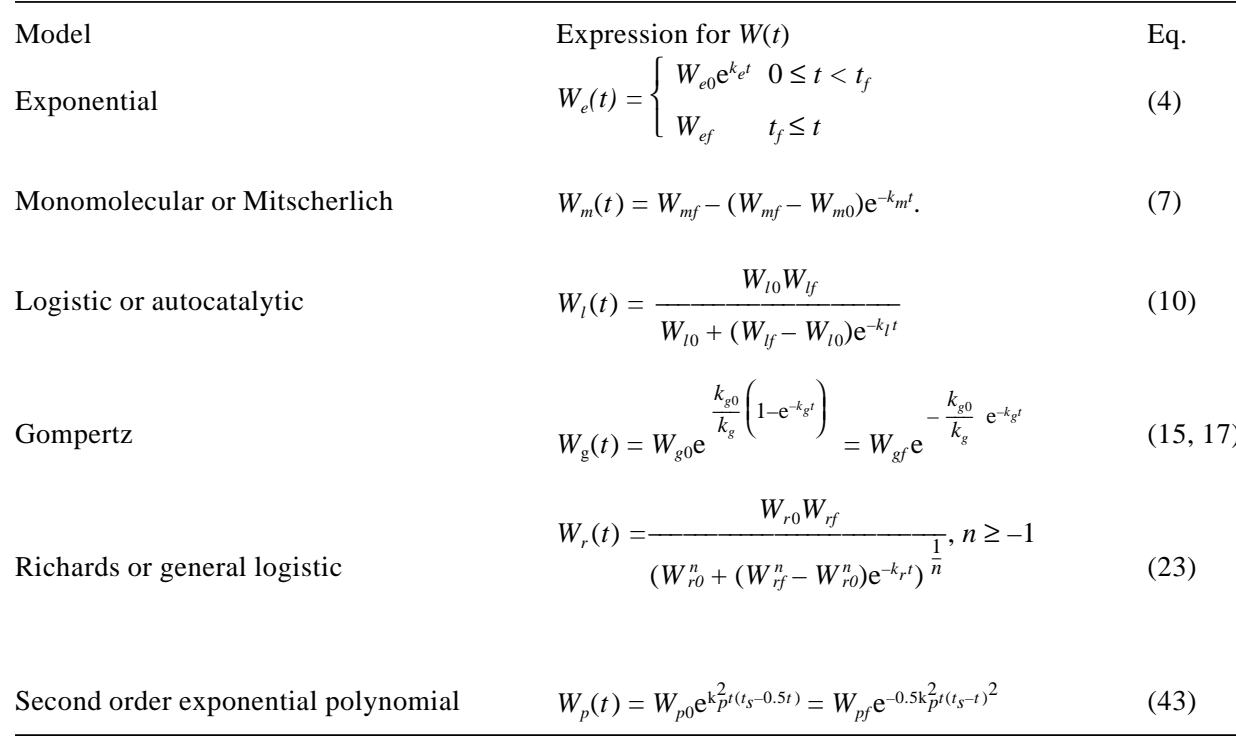

becomes depleted (c.f. Liebig's law of the minimum) (Figure 1). The growth rate is given by

$$
\frac{\mathrm{d} W_{e}}{\mathrm{~d} t}= \begin{cases}k_{e} W_{e} & 0 \leq t<t_{f} \\ 0 & t_{f} \leq t\end{cases}
$$

and the relative growth rate is given by

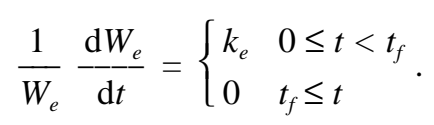

Integrating Eq. (2) yields (Figure 1)

$$
W_{e}(t)=\left\{\begin{array}{ll}
W_{e 0} \mathrm{e}^{k_{e} t} & 0 \leq t<t_{f} \\
W_{e f} & t_{f} \leq t
\end{array} .\right.
$$

From Eq. (4) expressions for $t_{f}$ or $k_{e}$ can be derived.

\section{Monomolecular or Mitscherlich growth}

The mathematical representation of monomolecular growth is borrowed from physical-chemistry, where it describes a first order irreversible chemical reaction. In plant nutrition and soil fertility it is also known as the Mitscherlich growth (Mitscherlich, 


\section{HEINEN}

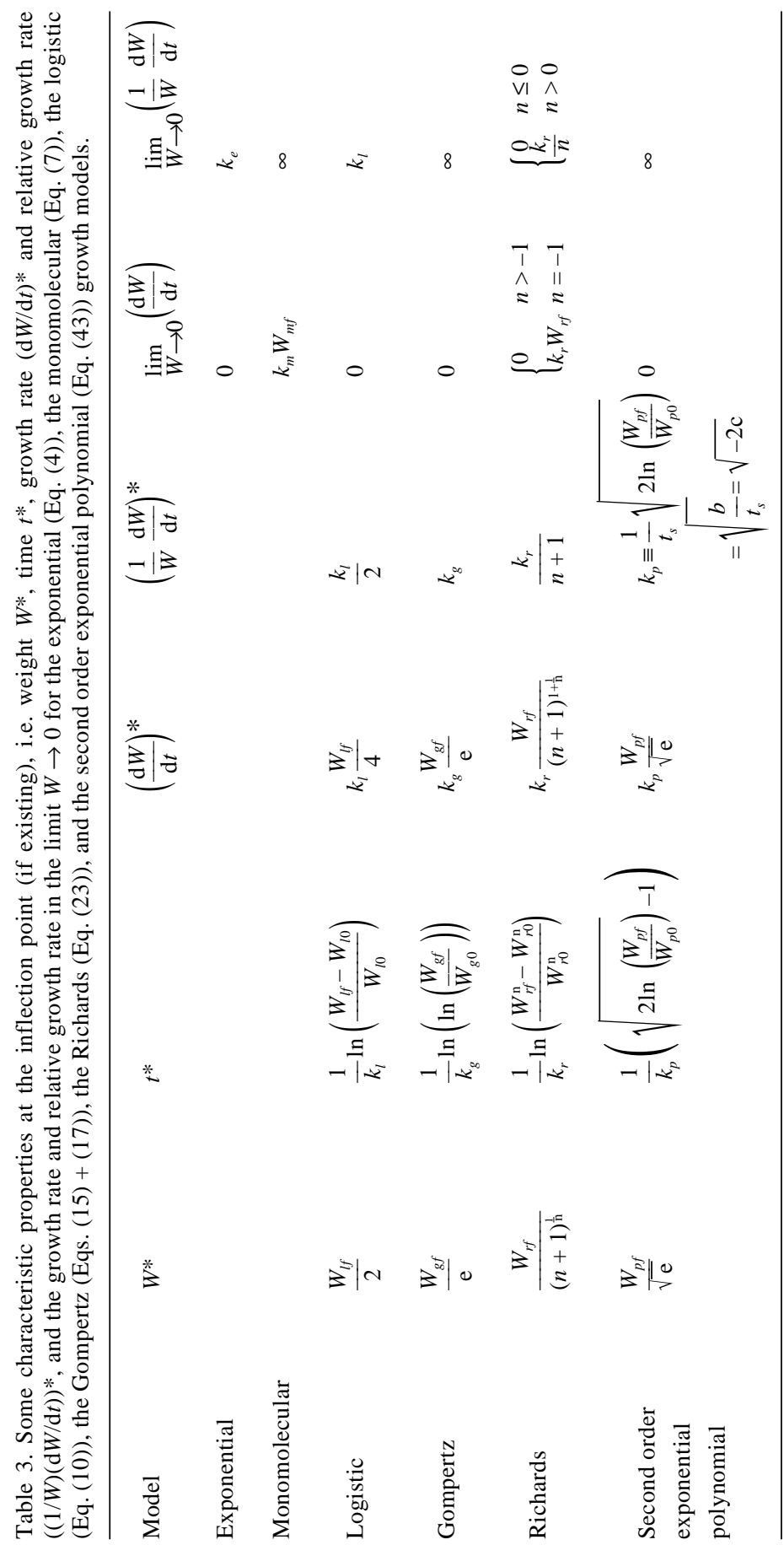


1928). Growth is irreversible, and the growth rate is proportional to the difference between the asymptotic maximum plant mass $W_{m f}$ and the plant mass already formed $W_{m}$ according to (Figure 2)

$$
\frac{\mathrm{d} W_{m}}{\mathrm{~d} t}=k_{m}\left(W_{m f}-W_{m}\right)
$$

and the relative growth rate is given by

$$
\frac{1}{W_{m}} \underset{\mathrm{d} t}{\mathrm{~d} W_{m}}=k_{m}\left(\frac{W_{m f}}{W_{m}}-1\right) \text {. }
$$

Integrating Eq. (5) yields (Figure 2)

$$
W_{m}(t)=W_{m f}-\left(W_{m f}-W_{m 0}\right) \mathrm{e}^{-k_{m} t}
$$

The proportionality constant $k_{m}$ can be expressed in terms of the parameter $W_{m f}$ (Table 3).

\section{Logistic or autocatalytic growth}

The growth rate of the logistic growth model is a combination of the growth rates of
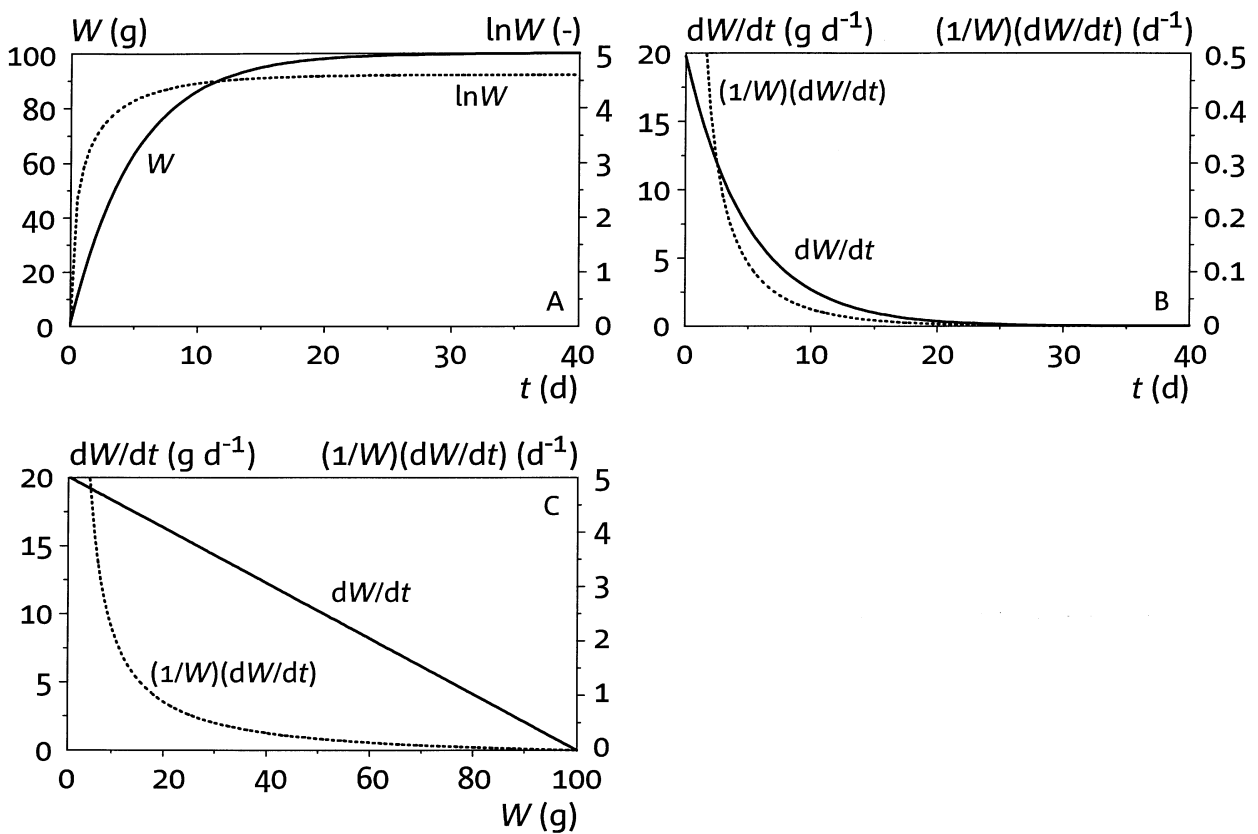

Figure 2. Monomolecular or Mitscherlich growth: (A) $W_{m}$ and $\ln W_{m}$ as a function of $t$, (B) $\mathrm{d} W_{m} / \mathrm{d} t$ and $\left(1 / W_{m}\right)\left(\mathrm{d} W_{m} / \mathrm{d} t\right)$ as a function of $t$, and (C) as a function of $W_{m}$; with $W_{m 0}=1 \mathrm{~g}, W_{m f}=100 \mathrm{~g}, k_{m}=0.2 \mathrm{~d}^{-1}$. 


\section{HEINEN}

the exponential and the monomolecular growth models. The growth rate is irreversible and it is proportional to $W_{l}$ and the difference between the asymptotic maximum $W_{l f}$ and $W_{l}$ (Figure 3):

$$
\frac{\mathrm{d} W_{l}}{\mathrm{~d} t}=k_{l}^{\prime} W_{l}\left(W_{l f}-W_{l}\right)=k_{l} W_{l}\left(1-\frac{W_{l}}{W_{l f}}\right) \text {. }
$$

The relative growth rate is given by

$$
\frac{1}{W_{l}} \frac{\mathrm{d} W_{l}}{\mathrm{~d} t}=k_{l}^{\prime}\left(W_{l f}-W_{l}\right)=k_{l}\left(1-\frac{W_{l}}{W_{l f}}\right),
$$

where $k_{l}=k_{l}^{\prime} / W_{l f}$. Integrating Eq. (8) (see Appendix A) yields (Figure 3)

$$
W_{l}(t)=\frac{W_{l 0} W_{l f}}{W_{l 0}+\left(W_{l f}-W_{l 0}\right) \mathrm{e}^{-k_{l} t}} .
$$

Logistic growth has an inflection point (Table 3). From Eqs. (8) or (9) with $W_{l}=W_{l}^{*}$ it follows that $k_{l}$ is a measure for the (relative) growth rate at the point of inflection (Table 3).
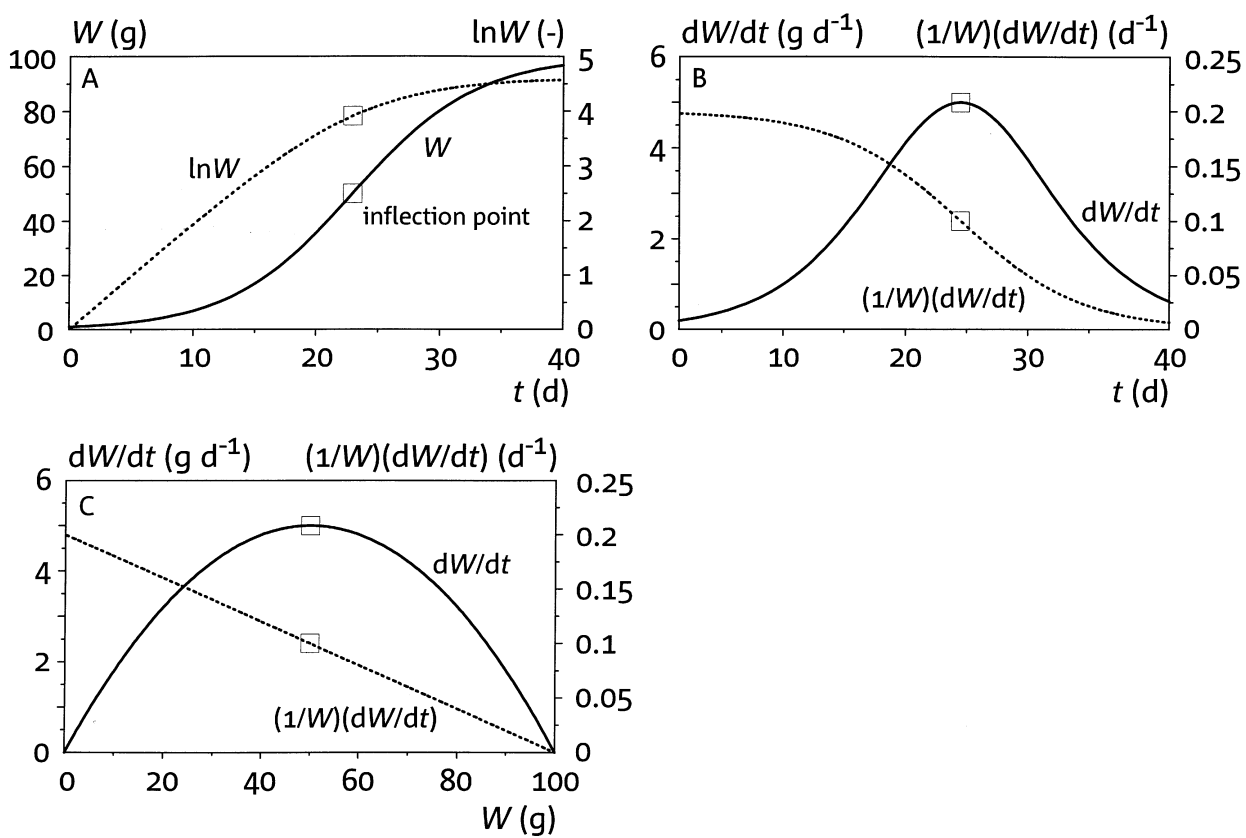

Figure 3. Logistic growth: (A) $W_{l}$ and $\ln W_{l}$ as a function of $t,(\mathrm{~B}) \mathrm{d} W_{l} / \mathrm{d} t$ and $\left(1 / W_{l}\right)\left(\mathrm{d} W_{l} / \mathrm{d} t\right)$ as a function of $t$, and (C) as a function of $W_{l}$; with $W_{l 0}=1 \mathrm{~g}, W_{l f}=100 \mathrm{~g}, k_{l}=0.2 \mathrm{~d}^{-1}$. 


\section{Gompertz growth}

B. Gompertz introduced in 1825 (as mentioned by Hunt, 1982) a growth model, in which the growth factors are non-limiting. The growth rate is proportional to $W_{g}$ and the relative growth rate decreases with time according to first-order kinetics (exponential decrease)

$$
\frac{\mathrm{d} W_{g}}{\mathrm{~d} t}=k_{g}^{\prime} W_{g}
$$

where $k_{g}^{\prime}$ decreases exponentially in time (e.g., due to aging or differentiation) according to

$$
\frac{\mathrm{d} k_{g}^{\prime}}{-\frac{\mathrm{d} t}{t}}=-k_{g} k_{g}^{\prime}
$$

where $k_{g}$ is a proportionality constant $\left(\mathrm{T}^{-1}\right)$. The result of integrating Eq. (12) between the limits $t=0, k_{g}{ }^{\prime}=k_{g 0}$ and $t=t, k_{g}{ }^{\prime}=k_{g}{ }^{\prime}$ introduced in Eq. (11) yields (Figure 4)

$$
\frac{\mathrm{d} W_{g}}{\mathrm{~d} t}=k_{g 0} W_{g} \mathrm{e}^{-k_{g} t}
$$

and the relative growth rate is given by
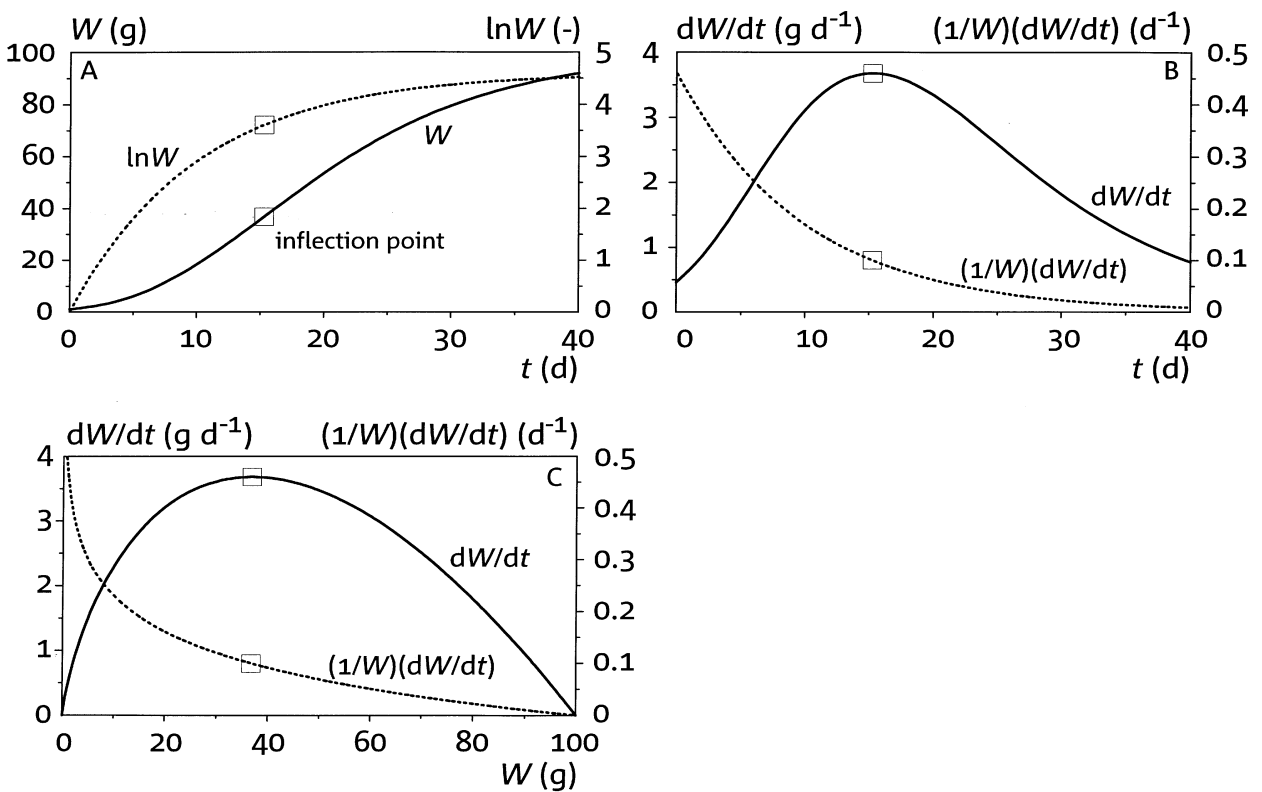

Figure 4. Gompertz growth: (A) $W_{g}$ and $\ln W_{g}$ as a function of $t$, (B) $\mathrm{d} W_{g} / \mathrm{d} t$ and $\left(1 / W_{g}\right)\left(\mathrm{d} W_{g} / \mathrm{d} t\right)$ as a function of $t$, and (C) as a function of $W_{g}$; with $W_{g 0}=1 \mathrm{~g}, W_{g f}=100 \mathrm{~g}, k_{g}=0.1 \mathrm{~d}^{-1}$. 


$$
\frac{1}{W_{g}} \frac{\mathrm{d} W_{g}}{\mathrm{~d} t}=k_{g 0} \mathrm{e}^{-k_{g} t}
$$

Integrating Eq. (13) yields (Figure 4)

$$
W_{\mathrm{g}}(t)=W_{g 0} \mathrm{e}^{\frac{k_{g 0}}{k_{g}}\left(1-\mathrm{e}^{-k_{g} t}\right)} .
$$

Since for $t \rightarrow \infty, W_{g}=W_{g f}$ it follows that

$$
W_{\mathrm{g} f}=W_{g 0} \mathrm{e}^{\frac{k_{g 0}}{k_{g}}} \text {. }
$$

Introducing Eq. (16) into Eq. (15) yields (Figure 4)

$$
W_{\mathrm{g}}(t)=W_{g f} \mathrm{e}^{-\frac{k_{g 0}}{k_{g}} \mathrm{e}^{-k_{g} t}} .
$$

Equation (17) can also be obtained directly by integrating Eq. (13) between the limits $t=t, W_{g}=W_{g}$ and $t=t_{f} \rightarrow \infty, W_{g}=W_{g f}$. From Eq. (16) it follows that

$$
k_{g 0}=k_{g} \ln \left(\frac{W_{g f}}{W_{g 0}}\right) \text {. }
$$

Equation (13) is still not of the form of Eq. (1), i.e. a function of $W_{g}$ only, because time $t$ is still included. This can be obtained by extracting an expression for $\exp \left(-k_{g} t\right)$ from Eq. (15) and substituting this result into Eq. (13) yielding (without or with using Eq. (16); Figure 4)

$$
\frac{\mathrm{d} W_{g}}{\mathrm{~d} t}=W_{g}\left(k_{g 0}-k_{g} \ln \left(\frac{W_{g}}{W_{g 0}}\right)\right)=k_{g} W_{g} \ln \left(\frac{W_{g f}}{W_{g}}\right),
$$

and the relative growth rate is given by

$$
\frac{1}{W_{g}} \frac{\mathrm{d} W_{g}}{\mathrm{~d} t}=k_{g 0}-k_{g} \ln \left(\frac{W_{g}}{W_{g 0}}\right)=k_{g} \ln \left(\frac{W_{g f}}{W_{g}}\right) \text {. }
$$

Gompertz growth has an inflection point (Table 3). From Eqs. (19) or (20) with $W_{g}=$ $W_{g}{ }^{*}$ it follows that $k_{g}$ is a measure for the (relative) growth rate at the inflection point (Table 3).

\section{Richards or general logistic growth}

Richards (1959) introduced a generalization of the above mentioned logistic model (Figure 5) 

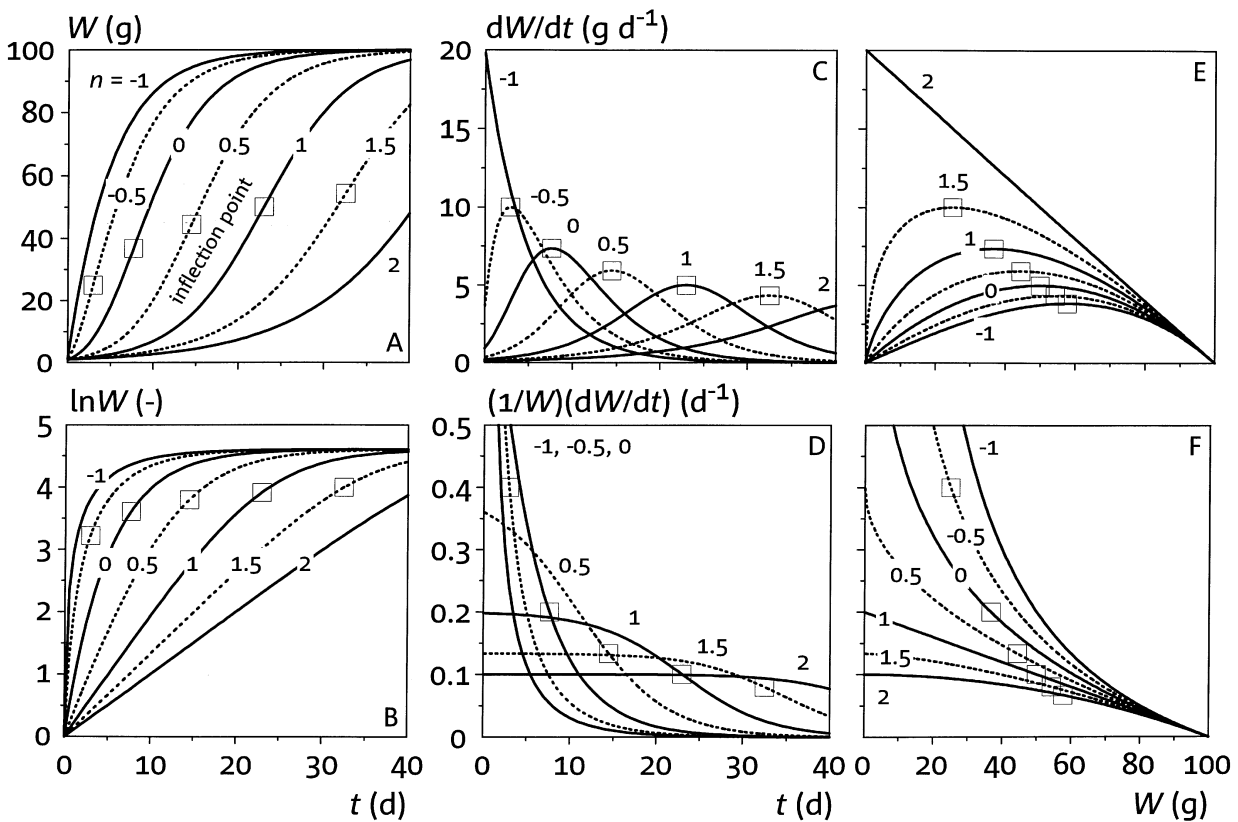

Figure 5. Richards or general logistic growth: (A) $W_{r}$, (B) $\ln W_{r},(\mathrm{C}) \mathrm{d} W_{r} / \mathrm{d} t$, and (D) $\left(1 / W_{r}\right)\left(\mathrm{d} W_{r} / \mathrm{d} t\right)$ as a function of $t$, and $(\mathrm{E}) \mathrm{d} W_{r} / \mathrm{d} t$ and $(\mathrm{F})\left(1 / W_{r}\right)\left(\mathrm{d} W_{r} / \mathrm{d} t\right)$ as a function of $W_{r}$; with $W_{r 0}=1 \mathrm{~g}, W_{r f}=100 \mathrm{~g}, k_{r}=$ $0.2 \mathrm{~d}^{-1}$ for several values of the dimensionless $n$ parameter: $n=-1,-0.5,0,0.5,1,1.5,2$.

$$
\frac{\mathrm{d} W_{r}}{\mathrm{~d} t}=k_{r} W_{r} \frac{W_{r f}^{n}-W_{r}^{n}}{n W_{r f}^{n}}=\frac{k_{r}}{n} W_{r}\left(1-\left(\frac{W_{r}}{W_{r f}}\right)^{n}\right),
$$

and the relative growth rate is given by

$$
\frac{1}{W_{r}} \frac{\mathrm{d} W_{r}}{\mathrm{~d} t}=k_{r} \frac{W_{r f}^{n}-W_{r}^{n}}{n W_{r f}^{n}}=\frac{k_{r}}{n}\left(1-\left(\frac{W_{r}}{W_{r f}}\right)^{n}\right),
$$

where the dimensionless constant $n$ must be larger than -1 , since then the growth rate in the limit $W_{r} \rightarrow 0$ is finite (see below). Integrating Eq. (21) (see Appendix A) (Figure 5) yields

$$
W_{r}(t)=\frac{W_{r 0} W_{r f}}{\left(W_{r 0}^{n}+\left(W_{r f}^{n}-W_{r 0}^{n}\right) \mathrm{e}^{-k_{r} t}\right)^{\frac{1}{n}}}, n \geq-1 .
$$

The Richards growth equation includes some special cases. For $n=1$ Eq. (23) reduces to the logistic model (Eq. (10)). Substituting $n=-1$ in Eq. (23) and using simple algebra one obtains the monomolecular or Mitscherlich growth curve (Eq. (7)). For $n=0$ it represents the Gompertz model, as is shown next. By taking the limit $n \rightarrow 0$ of $\mathrm{d} W_{r} / \mathrm{d} t$ and by using the expansion series for $x^{n}$ (with $x=W_{r} / W_{r f}$ ), it can be shown that this limit corresponds to Eq. (19): 


\section{HEINEN}

$$
\lim _{n \rightarrow 0} \frac{\mathrm{d} W_{r}}{\mathrm{~d} t}=\lim _{n \rightarrow 0} k_{r} W_{r}\left(\frac{1}{n}-\frac{1}{n}\left(\frac{W_{r}}{W_{r f}}\right)^{n}\right) .
$$

The expansion for $x^{n}$ is

$$
x^{n}=\mathrm{e}^{n \ln x}=1+\frac{n \ln x}{1 !}+\frac{(n \ln x)^{2}}{2 !}+\frac{(n \ln x)^{3}}{3 !}+\ldots
$$

so that Eq. (24) can be written analogous to Eq. (19), i.e.,

$$
\lim _{n \rightarrow 0} \frac{\mathrm{d} W_{r}}{\mathrm{~d} t}=-k_{r} W_{r}\left(\ln \left(\frac{W_{r}}{W_{r f}}\right)+\frac{n}{2 !}\left(\ln \left(\frac{W_{r}}{W_{r f}}\right)\right)^{2}+\frac{n}{3 !}\left(\ln \left(\frac{W_{r}}{W_{r f}}\right)\right)^{3}+\ldots\right)=k_{r} W_{r} \ln \left(\frac{W_{r f}}{W_{r}}\right) .
$$

The Richards model has an inflection point (Table 3; Figure $6 ; n>-1$ ). For $n=1$ and $n=0$ the inflection points of the logistic and the Gompertz model are obtained. For $n>-1$, the limit $W_{r} \rightarrow 0$ of $\mathrm{d} W_{r} / \mathrm{d} t$ equals

$$
\lim _{W_{r} \rightarrow 0} \frac{\mathrm{d} W_{r}}{\mathrm{~d} t}=-\frac{k_{r}}{n W_{r f}^{n}} \lim _{W_{r \rightarrow 0}} W_{r}^{n+1}=0, n>-1 \text {. }
$$

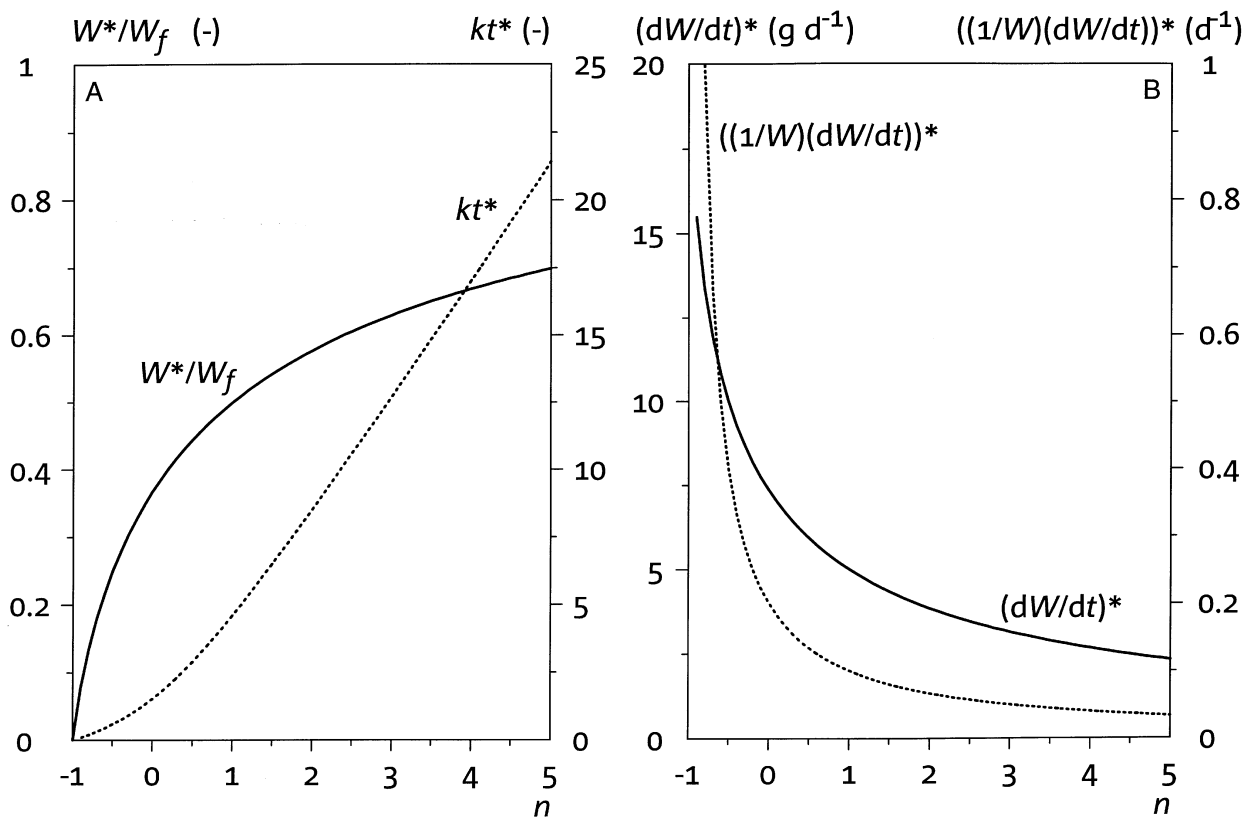

Figure 6. (A) The normalized plant mass at inflection point, $W_{r} * / W_{r f}$, and the normalized time of inflection, $k_{r} t_{r}{ }^{*}$, as a function of $n$, and (B) the growth rate, $\left(\mathrm{d} W_{r} / \mathrm{d} t\right)^{*}$, and the relative growth rate, $\left(\left(1 / W_{r}\right)\left(\mathrm{d} W_{r} / \mathrm{d} t\right)\right)^{*}$, at inflection point as a function of the dimensionless $n$ parameter in the Richards growth model. 
For $n<-1$ this limit is undefined, and for $n=-1$ this limit yields $k_{r} W_{r f}$ (monomolecular growth).

From Eqs. (21) or (22) with $W_{r}=W_{r}^{*}$ it follows that $k_{r}$ is a measure for the (relative) growth rate at the inflection point (Table 3 ).

\section{Exponential polynomial growth}

Exponential polynomials do not have any physiological meaning from which they can be derived. Only afterwards the parameters of these polynomials can be given a physiological meaning. The general form of exponential polynomials equals

$$
W_{p}(t)=\mathrm{e}^{a+b t+c t^{2}+d t^{3}+\ldots} .
$$

The first order exponential polynomial reads

$$
W_{p}(t)=\mathrm{e}^{a+b t}=a^{\prime} \mathrm{e}^{b t},
$$

where $a^{\prime}=\mathrm{e}^{a}$. Equation (29) is analogous to exponential growth (cf. Eq. (4)).

The second order exponential polynomial reads (Figure 7)

$$
W_{p}(t)=\mathrm{e}^{a+b t+c t^{2}}
$$
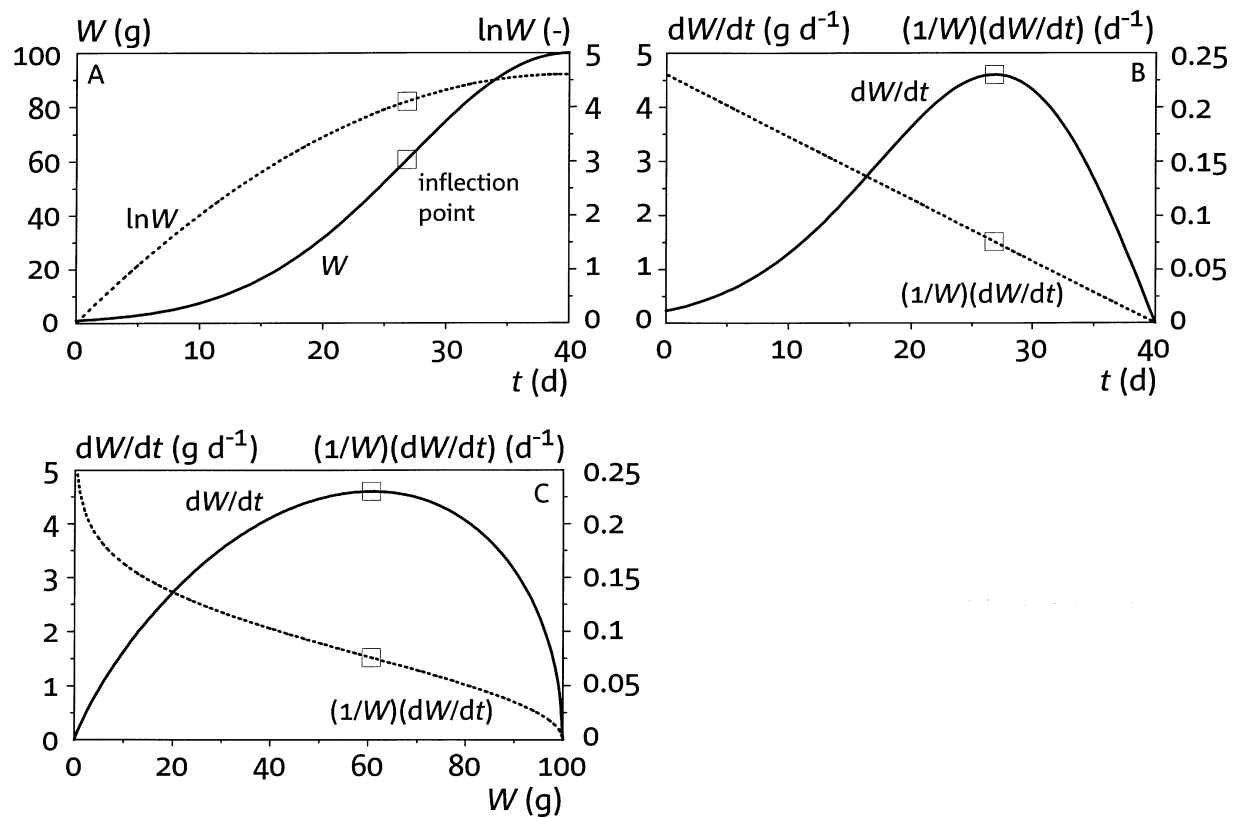

Figure 7. Second order exponential polynomial growth: (A) $W_{p}$ and $\ln W_{p}$ as a function of $t$, (B) $\mathrm{d} W_{p} / \mathrm{d} t$ and $\left(1 / W_{p}\right)\left(\mathrm{d} W_{p} / \mathrm{d} t\right)$ as a function of $t$, and (C) as a function of $W_{p}$; with $W_{p 0}=1 \mathrm{~g}, W_{p f}=100 \mathrm{~g}, t_{s}=40 \mathrm{~d}$, $k_{p}=0.075871 \mathrm{~d}^{-1}$. 


\section{HEINEN}

For $t=0$ the parameter $a$ is a measure for $W_{p 0}$ :

$$
a=\ln W_{p 0} .
$$

The form of the second order exponential polynomial depends on the values of $b$ and $c$. For our purposes $b>0$ and $c<0$ is the most interesting case. Equation (30) is symmetrical around $t=t_{s}$, with a positive growth rate between $t=0$ and $t=t_{s}$. This is the time interval which is considered, and we require $t_{s}$ to be the time at the end of the growth period at which $W_{p}=W_{p f}$. The growth rate is obtained by differentiating Eq. (30) with respect to $t$ yielding (Figure 7)

$$
\frac{\mathrm{d} W_{p}}{\mathrm{~d} t}=(b+2 c t) \mathrm{e}^{a+b t+c t^{2}}=(b+2 c t) W_{p},
$$

and the relative growth rate is given by

$$
\frac{1}{W_{p}} \stackrel{\mathrm{d} W_{p}}{-} \stackrel{-\underline{\mathrm{d}} t}{t}=(b+2 c t) .
$$

From Eq. (33) it can be seen that $b$ equals the relative growth rate at $t=0$. The maximum of the curve is obtained by solving $\mathrm{d} W_{p} / \mathrm{d} t=0$ for $t$ yielding $t_{s}$ :

$$
t_{s}=-\frac{b}{2 c}
$$

At $t_{s} W_{p}=W_{p f}$, and an expression for $W_{p f}$ follows from Eq. (30) using Eq. (34):

$$
W_{p f}=\mathrm{e}^{a-\frac{b^{2}}{4 c}}=W_{p 0} \mathrm{e}^{-\frac{b^{2}}{4 c}}
$$

From Eqs. (34) and (35) expressions for the two unknown parameters $b$ and $c$ in terms of the quantities $W_{p 0}, W_{p f}$ and $t_{s}$ can be derived as

$$
b=\frac{2}{t_{s}} \ln \left(\frac{W_{p f}}{W_{p 0}}\right) \text {, and } c=-\frac{1}{t_{s}^{2}} \ln \left(\frac{W_{p f}}{W_{p 0}}\right) .
$$

The inflection point inside the time interval $\left[0, t_{s}\right]$ occurs at (Table 3 )

$$
t_{p}^{*}=t_{s}-\sqrt{-\frac{1}{2 c}}=t_{s}\left(1-\sqrt{\frac{1}{2 \ln \left(\frac{W_{p f}}{W_{p 0}}\right)}}\right) .
$$

Evaluating Eqs. (30), (31), (36), and (37) at the point of inflection results in an expression for $W_{p}^{*}$ (Table 3). From Eqs. (34) and (37) it follows that $b$ and $c$ are measures for the difference between $t_{s}$ and $t_{p}^{*}$ : 


$$
b=\frac{t_{s}}{\left(t_{s}-t_{p}^{*}\right)^{2}}, \text { and } c=-\frac{1}{2\left(t_{s}-t_{p}^{*}\right)^{2}} .
$$

Parameters $b$ and $c$ can not be expressed in terms of $W_{p f}$ and $W_{p}^{*}$, since one can imagine that at different $\left(t_{s}-t_{p}^{*}\right)$ the same difference in $W_{p f}$ and $W_{p}^{*}$ can be obtained.

In Eq. (32) $\mathrm{d} W_{p} / \mathrm{d} t$ is not explicitly given as a function of $W_{p}$ only, because $t$ is included. From Eq. (30) an expression for $t$, within the interval $\left[0, t_{s}\right]$, can be obtained yielding (using Eq. (36))

$$
t=t_{s}\left(1-\sqrt{\left.\frac{\ln \left(\frac{W_{p f}}{W_{p}}\right)}{-\ln \left(\frac{W_{p f}}{W_{p 0}}\right)}\right)}\right) .
$$

Substituting Eq. (39) into Eq. (32), using Eq. (36), yields (Figure 7)

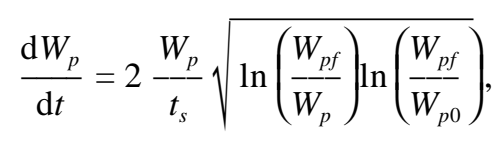

and the relative growth rate is given by

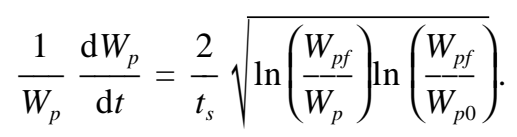

Since the logistic, Gompertz and Richards growth models have a proportionality constant $k$ which is a measure for the relative growth rate at the point of inflection, it is interesting to see if we can define a similar parameter $k_{p}$. We define $k_{p}$ as the relative growth rate at the inflection point. Evaluating Eq. (41) at the inflection point using the expression of $W^{*}$ (Table 3 ) yields

$$
k_{p}=\frac{1}{t_{s}} \sqrt{2 \ln \left(\begin{array}{l}
W_{p f} \\
\frac{W_{p 0}}{W_{p 0}}
\end{array}\right)}=\sqrt{\frac{b}{t_{s}}}=\sqrt{-2 c} .
$$

Now we can write expressions for $W_{p}(t), \mathrm{d} W_{p} / \mathrm{d} t$ and $\left(1 / W_{p}\right)\left(\mathrm{d} W_{p} / \mathrm{d} t\right)$ in terms of the parameters $W_{p 0}, W_{p f}$ and $k_{p}$. Substituting Eqs. (31), (36), and (42) in Eq. (30) yields

$$
W_{p}(t)=W_{p 0} \mathrm{e}^{k_{p}^{2} t\left(t_{s}-0.5 t\right)}=W_{p f} \mathrm{e}^{-0.5 k_{p}^{2}\left(t_{s}-t\right)^{2}} .
$$

Differentiating Eq. (43) to $t$, or substituting Eq. (42) in Eq. (40), yields

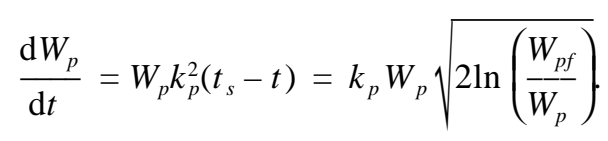




\section{HEINEN}

The relative growth rate follows from Eq. (44) as

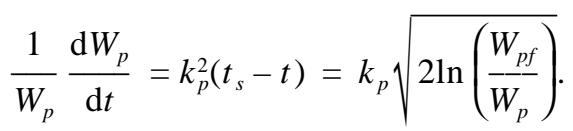

France and Thornley (1984) advise not to use higher order exponential polynomials, because of possible "overfitting" of the measured data.

\section{Equivalent expressions for the growth models}

The statistical processing program Genstat (Anon., 1993) uses equivalent expressions for the non-linear - in terms of the unknown parameters - growth equations presented above (as listed in Table 2). The exponential and monomolecular growth equations are both described by

$$
Y_{e m}(t)=a_{e m}+b_{e m} r^{t}
$$

The logistic growth equation is described by

$$
Y_{l}(t)=a_{l}+\frac{c_{l}}{1+\mathrm{e}^{-b_{l}\left(t-m_{l}\right)}},
$$

the Gompertz equation by

$$
Y_{g}(t)=a_{g}+c_{g} \mathrm{e}^{-e^{-b_{s}\left(t-m_{g}\right)}}
$$

and the Richards equation by

$$
Y_{l}(t)=a_{r}+\frac{c_{r}}{1+p \mathrm{e}^{-b r(t-m r) \frac{1}{p}}}
$$

The variable $Y$ represents the plant mass $W$. The parameters $a$ and $(a+c)$ in Eqs. (46) to (49) represent the lower and upper asymptotes, respectively. As in the previous section, $a$ will be absent in Eqs. (47)-(49). The parameters $b$ are measures of the slopes of the curves, the parameters $m$ represent the time of inflection, i.e. $m=t^{*}$, and $p$ and $r$ are dimensionless parameters. It is clear that for the Richards model $p=$ $n$. In Genstat's directive FITCURVE the choice of the model can be given as an option. Genstat determines estimators for the parameters $a, b, c, m, p$ and $r$ for given measured data. The relation between the unknown parameters in Eqs. (4), (7), (10), $(15)+(17)$ and (23) and the Genstat parameters will be given below. For convenience, the relationships are listed in Table 4.

The second order exponential polynomial, i.e. Eq. (30) or Eq. (43), can be fitted via linear regression after ln-transformation. 
Table 4. The relation between the parameters $k, W_{f}, W_{0} k_{g 0}$ and $n$ of the exponential (Eq. (4)), monomolecular (Eq. (7)), logistic (Eq. (10)), Gompertz (Eqs. (15) + (17)), Richards (Eq. (23)), and second order exponential polynomial (Eq. (43)) growth models, and the estimated parameters $a, b, c, m, r$, and $p$ of equivalent expressions by Genstat.

\begin{tabular}{|c|c|c|c|c|c|}
\hline Model & $k$ & $W_{f}$ & $W_{0}$ & $k_{g 0}$ & $n$ \\
\hline Exponential & $\ln r$ & & $b_{e m}$ & & \\
\hline Monomolecular & $\ln \frac{1}{r}$ & $a_{e m}$ & $a_{e m}+b_{e m}$ & & \\
\hline Logistic & $b_{l}$ & $c_{l}$ & $\frac{c_{l}}{1+\mathrm{e}^{b_{l} m_{l}}}$ & & \\
\hline Gompertz & $b_{g}$ & $c_{g}$ & $c_{g} \mathrm{e}^{-\mathrm{e}^{b_{g} m_{g}}}$ & $b_{g} \mathrm{e}^{b_{g} m_{g}}$ & \\
\hline Richards & $b_{r}$ & $c_{r}$ & $\frac{c_{r}}{\left(1+p \mathrm{e}^{b_{r} m_{r}}\right)^{\frac{1}{p}}}$ & & $p$ \\
\hline $\begin{array}{l}\text { Second order exponential } \\
\text { polynomial }\end{array}$ & $\sqrt{\frac{b}{t_{s}}}$, or $\sqrt{-2 \mathrm{c}}$ & $\mathrm{e}^{a-\frac{b^{2}}{4 c}}$ & $\mathrm{e}^{a}$ & & \\
\hline
\end{tabular}

\section{Exponential growth}

With the substitution $r=\exp \left(k_{e}\right)$ and the assumption $a_{e m}=0$, Eq. (46) becomes

$$
Y_{e m}(t)=b_{e m} \mathrm{e}^{k_{e} t}
$$

Equation (50) is equivalent to Eq. (4) for $b_{e m}=W_{e 0}$ and $\ln r=k_{e}$.

Monomolecular or Mitscherlich growth

With the substitution $r=\exp \left(-k_{m}\right)$ Eq. (46) becomes

$$
Y_{e m}(t)=a_{e m}+b_{e m} \mathrm{e}^{-k_{m} t}
$$

Equation (51) is equivalent to Eq. (7) for $a_{e m}=W_{m f}, a_{e m}+b_{e m}=W_{m 0}$ and $\ln (1 / r)=k_{m}$. In Genstat it is possible that the program uses the restriction $r>1$. This yields a negative value for $a_{e m}$, and this means that $W_{m f}$ becomes negative, which is impossible. In that case Genstat assumes exponential growth.

\section{Logistic or autocatalytic growth}

In Eq. (10) $W_{l f}$ is the asymptotic maximum for $W_{l}$, which is represented by $c_{l}$ in Eq. (47), i.e. $c_{l}=W_{l f}$. With $b_{l}=k_{l}$ and $m_{l}=t_{l}{ }^{*}$, and using the definition of $t_{l}^{*}$ (Table 3) Eq. (47) becomes

$$
Y_{l}(t)=\frac{W_{l f}}{1+\mathrm{e}^{k_{l} t} t_{l}^{*} \mathrm{e}^{-k_{l} t}}=\frac{W_{l 0} W_{l f}}{W_{l 0}+\left(W_{l f}-W_{l 0}\right) \mathrm{e}^{-k_{l} t}} .
$$




\section{HEINEN}

Under the given conditions, Eq. (47) is equivalent to Eq. (10) (= Eq. (52)). $W_{l 0}$ follows from the definition of $t_{l}^{*}$ (Table 3 ) and the given conditions:

$$
W_{l 0}=\frac{c_{l}}{1+\mathrm{e}^{b_{l} m_{l}}} .
$$

\section{Gompertz growth}

In Eq. (17) $W_{g f}$ is the asymptotic maximum for $W_{g}$, which is represented by $c_{g}$ in Eq. (48), i.e. $c_{g}=W_{g f}$. With $b_{g}=k_{g}$ and $m_{g}=t_{g}{ }^{*}$, and using the definition of $t_{g}{ }^{*}$ (Table 3) Eq. (48) becomes

$$
Y_{\mathrm{g}}(t)=W_{g f}-e^{k_{g} t_{g}^{*} e^{-k_{g} t}}=W_{g f} \mathrm{e}^{-\frac{k_{g o}}{k_{g}} \mathrm{e}^{-k_{g} t}} .
$$

Under the given conditions, Eq. (48) is equivalent to Eq. (17) (= Eq. (54)). $k_{\mathrm{g} 0}$ follows from the definition of $t_{g} *$ (Table 3 ) and the given conditions:

$$
k_{g 0}=b_{g} \mathrm{e}^{b_{g} m_{g}} .
$$

$W_{g 0}$ follows from Eq. (16) and Eq. (55) under the given conditions:

$$
W_{g 0}=c_{g} \mathrm{e}^{-e^{b_{s} m_{g}}} .
$$

\section{Richards or general logistic growth}

In Eq. (23) $W_{r f}$ is the asymptotic maximum for $W_{r}$, which is represented by $c_{r}$ in Eq. (49), i.e. $c_{r}=W_{r f}$. With $p=n, b_{r}=k_{r}$ and $m_{r}=t_{r}{ }^{*}$, and using the definition of $t_{r}{ }^{*}$ (Table 3) Eq. (49) becomes

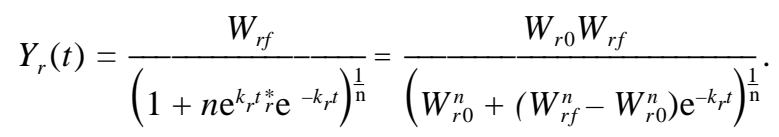

Under the given conditions, Eq. (49) is equivalent to Eq. (23) (= Eq. (57)). $W_{r 0}$ follows from the definition of $t_{r}^{*}$ (Table 3 ) and the given conditions:

$$
W_{r 0}=\frac{c_{r}}{\left(1+\mathrm{e}^{b_{r} m_{r}}\right)^{\frac{1}{\mathrm{n}}}} .
$$

\section{Exponential polynomial growth}

The parameters of the second order exponential polynomial cannot be estimated directly using standard fit-procedures in Genstat (Anon., 1993). After ln-transforming Eq. (30) a linear equation - in terms of the parameters - is obtained

$$
\ln W_{p}=a+b t+c t^{2} .
$$


Equation (59) can be solved directly using linear regression giving estimates of $a, b$ and $c$. A disadvantage of the ln-transformation method is that the fitted curve may show its optimum within the measurement interval $\left[0, t_{m i}\right]$ (the lower $W_{p}$ values "pull stronger" at the curve to be fitted), with $t_{m i}$ the last time of the measurement interval. For the exponential polynomial (as described above) we assumed that the maximum occurs at the end of the growth period, i.e. at $t=t_{s}=t_{m i}$. The method of transformation determines the place of this optimum. Therefore, it is advised to transform the data as follows

$$
W_{p, t}=\ln \left(W_{p}(t)+\varepsilon\right), \varepsilon>0 .
$$

In that case the optimum is a function of the constant $\varepsilon$. It is proposed that in case the data show a $\mathrm{S}$-shape-like distribution, $\varepsilon$ is chosen such that the optimum occurs at $t=t_{s}=t_{m i}$. That means that the parameters $b$ and $c$ are restricted according to Eq. (34).

\section{Example}

To demonstrate the use of the models described above, the dry weight data of lettuce heads of Heinen et al. (1991) were fitted to the logistic, Gompertz, Richards, and second order polynomial growth equations (Figure 8A). It goes beyond the scope of this report to discuss the observations, since similar discussions are given in Heinen et al. (1991). The estimated parameters of the logistic, Eq. (47), Gompertz, Eq. (48), Richards, Eq. (49), and second order exponential polynomial, Eq. (60), models were used to calculate the parameters in Eqs. (10), (15) + (17), (23), and (43) respectively, according to Table 4 (Table 5). The value of $\varepsilon$ in Eq. (60) was iteratively estimated as 0.05823 , in which case the optimum of the exponential curve occurs at the last time of the measurement interval. Similarly, the cumulative uptake by the lettuce heads of $\mathrm{N}, \mathrm{P}$ and $\mathrm{K}$ were fitted to the logistic model (Table 5). These fitted data were scaled by dividing them by the fitted maximum value $W_{f}$ (Figure $8 \mathrm{~B}$ ).

The question remains which of the functions is the best. The answer will depend on the researcher and his/hers interests and ideas about the growth of the crop. Judgement of a good fit is mostly done based on high correlation coefficient $r^{2}$ combined with a visually good fit, and the choice of the function with the least number of parameters or the function that comes closest to the concept of growth used by the researcher. Here, one could say that the logistic function is preferred: highest $r^{2}$ and the least number of parameters. It is recognized that the fit is biased by measurement errors. For the data used in this example no measurement errors are known, since data refer to a single sample obtained from several plants. Moreover, if the crop has distinct growth stages, such as vegetative growth and flowering, for each growth stage a growth function may be used. For example, Hammond and Kirkham (1949) used exponential growth functions for three growth periods of soybeans and four growth periods of corn. 


\section{HEINEN}
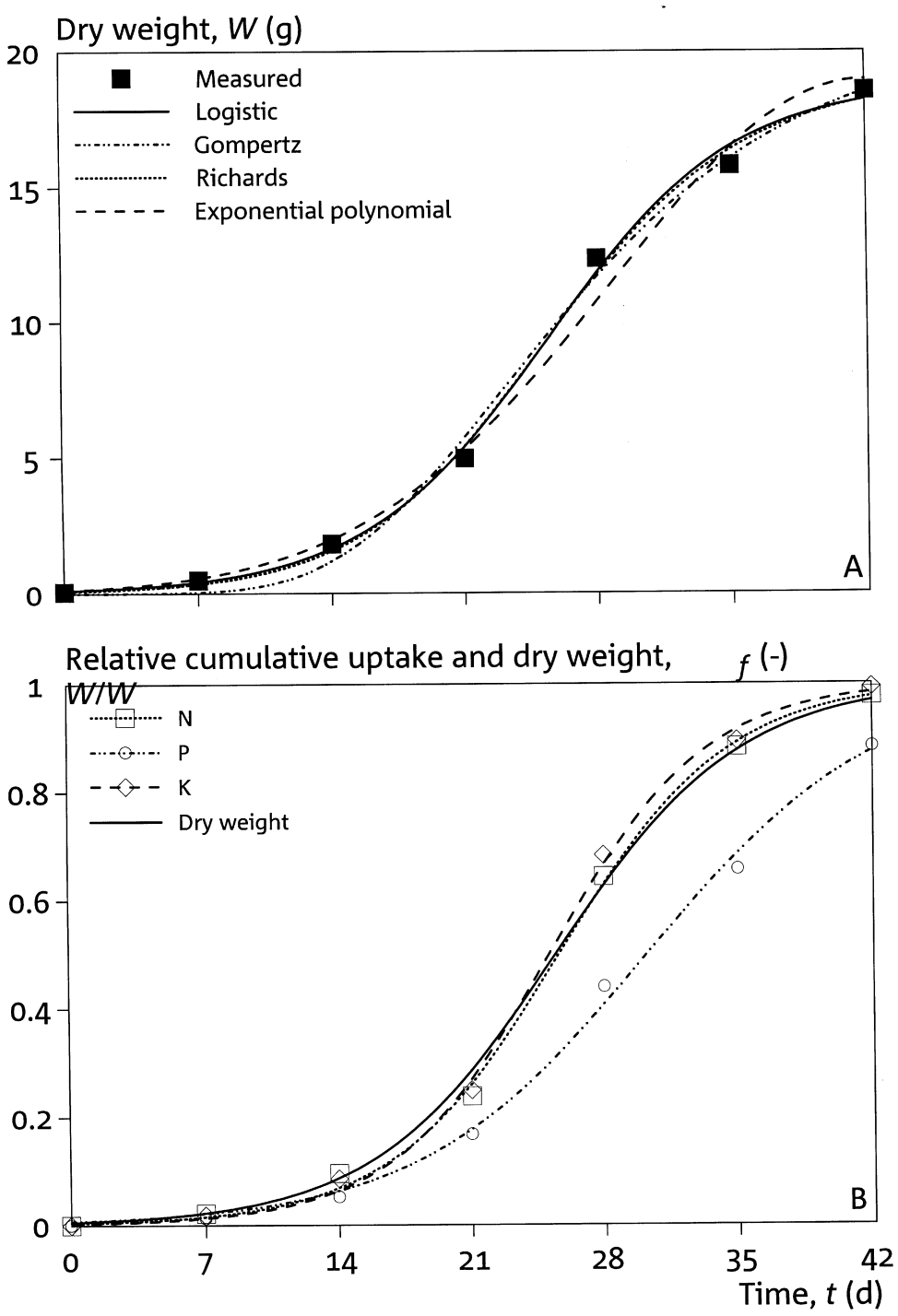

Figure 8. (A) Dry weight data of lettuce head from Heinen et al. (1991) fitted to the logistic, Gompertz, Richards and second order exponential polynomial models. (B) Cumulative uptake data of N, P and K by lettuce head from Heinen et al. (1991) fitted to the logistic model.

\section{One model fits all}

As was mentioned before, the Richards growth model includes the logistic, the monomolecular or Mitscherlich, and the Gompertz growth equations. In fact, these four models and the exponential growth equation can be represented by the following family of equations, which is analogous to Eq. (46) (Hunt, 1982) 
Table 5. The estimated and calculated parameters (PM) of the logistic (L, Eq. (10)), Gompertz (G, Eqs. $(15)+(17)$ ), Richards (R, Eq. (23)) and second order exponential polynomial (EP, Eq. (30) or (43)) models for the dry weight data ( $\mathrm{g}$ per lettuce head), and the estimated and calculated parameters for the cumulative uptake data (mmol per lettuce head) of nitrogen $(\mathrm{N})$, phosphorus $(\mathrm{P})$ and potassium $(\mathrm{K})$ for the logistic model. The correlation coefficient is given as by $r^{2}$. For the estimated parameters the standard errors, as given by Genstat, are listed between brackets.

\begin{tabular}{|c|c|c|c|c|c|c|c|}
\hline \multirow[t]{2}{*}{ PM } & \multicolumn{4}{|l|}{$\mathrm{L}$} & \multirow{2}{*}{$\frac{\mathrm{G}}{\mathrm{dry}}$} & \multirow{2}{*}{$\frac{\mathrm{R}}{\mathrm{dry}}$} & \multirow{2}{*}{$\begin{array}{c}\text { EP } \\
\text { dry }\end{array}$} \\
\hline & dry & $\mathrm{N}$ & $\mathrm{P}$ & $\mathrm{K}$ & & & \\
\hline$W_{0}(\mathrm{~g}$ or $\mathrm{mmol})$ & 0.100 & 0.218 & 0.044 & 0.111 & 0.0 & 0.067 & 0.115 \\
\hline$W_{f}(\mathrm{~g}$ or $\mathrm{mmol})$ & $\begin{array}{l}18.78 \\
(0.669)\end{array}$ & $\begin{array}{c}68.97 \\
(1.60)\end{array}$ & $\begin{array}{c}6.65 \\
(0.422)\end{array}$ & $\begin{array}{c}47.46 \\
(1.05)\end{array}$ & $\begin{array}{c}20.81 \\
(1.55)\end{array}$ & $\begin{array}{c}19.04 \\
(1.25)\end{array}$ & 18.94 \\
\hline$k \quad\left(\mathrm{~d}^{-1}\right)$ & $\begin{array}{c}0.2054 \\
(0.0208)\end{array}$ & $\begin{array}{c}0.2244 \\
(0.0160)\end{array}$ & $\begin{array}{c}0.1660 \\
(0.0178)\end{array}$ & $\begin{array}{c}0.2409 \\
(0.0178)\end{array}$ & $\begin{array}{c}0.1145 \\
(0.0189)\end{array}$ & $\begin{array}{c}0.1854 \\
(0.0716)\end{array}$ & 0.0761 \\
\hline$t^{*}(\mathrm{~d})$ & $\begin{array}{l}25.47 \\
(0.646)\end{array}$ & $\begin{array}{l}25.63 \\
(0.407)\end{array}$ & $\begin{array}{l}30.16 \\
(1.10)\end{array}$ & $\begin{array}{l}25.14 \\
(0.387)\end{array}$ & $\begin{array}{l}23.24 \\
(0.972)\end{array}$ & $\begin{array}{l}25.12 \\
(1.65)\end{array}$ & 28.86 \\
\hline$n$ & & & & & & $\begin{array}{c}0.785 \\
(0.761)\end{array}$ & \\
\hline$k_{g 0}\left(\mathrm{~d}^{-1}\right)$ & & & & & 1.6396 & & \\
\hline$a$ & & & & & & & $\begin{array}{l}-2.1661 \\
(0.0742)\end{array}$ \\
\hline$b \quad\left(\mathrm{~d}^{-1}\right)$ & & & & & & & $\begin{array}{c}0.24319 \\
(0.00827)\end{array}$ \\
\hline$c\left(\mathrm{~d}^{-2}\right)$ & & & & & & & $\begin{array}{c}-0.002895 \\
(0.000189)\end{array}$ \\
\hline$\varepsilon$ & & & & & & & 0.05823 \\
\hline$r^{2}$ & 99.9 & 99.9 & 99.8 & 99.9 & 99.9 & 99.8 & 99.9 \\
\hline
\end{tabular}

$$
Y=\alpha+\beta \gamma^{t}
$$

where $Y$ is a function of $W$, and the parameters $\alpha, \beta$ and $\gamma$ depend on the kind of growth model under consideration (Table 6).

For completeness, it is mentioned here that in Genstat a procedure is available called FITSCHNUTE. It can be used to fit data according to the growth model of Schnute (1981). It is available in the CBW-DLO Genstat-procedures-library which can be downloaded from the Internet (visit www.cpro.dlo.nl/cbw; CBW stands for Centre for Biometry Wageningen and DLO stands for the Dutch Agricultural Research Organization). The procedure is described in detail by Keen (1988). The model reads

$$
Y=y+\sigma
$$

where $y$ is a function of $t$ according to

$$
Y(t)=\left(y_{1}^{\mu}+\left(y_{2}^{\mu}-y_{1}^{\mu}\right) \frac{1-\mathrm{e}^{-v\left(t-t_{1}\right)}}{1-\mathrm{e}^{-v\left(t_{2}-t_{1}\right)}}\right)^{\frac{1}{\mu}} .
$$




\section{HEINEN}

Table 6. Expressions for the parameters $y, \alpha, \beta$ and $\gamma$ of Eq. (61) to obtain the exponential, the monomolecular, the logistic, the Gompertz and the Richards growth models.

\begin{tabular}{lcccc}
\hline Model & $y$ & $\alpha$ & $\beta$ & $\gamma$ \\
Exponential & $W_{e}$ & 0 & $W_{e 0}$ & $\mathrm{e}^{k_{e}}$ \\
Monomolecular & $W_{m}$ & $W_{m f}$ & $W_{m 0}-W_{m f}$ & $\mathrm{e}^{-k_{m}}$ \\
Logistic & $W_{l}^{-1}$ & $W_{l f}^{-1}$ & $W_{l 0}^{-1}-W_{l f}^{-1}$ & $\mathrm{e}^{-k_{l}}$ \\
Gompertz & $\ln W_{g}$ & $\ln W_{g f}$ & $\ln \frac{W_{g f}}{W_{g 0}}=-\frac{k_{g 0}}{k_{g}}$ & $\mathrm{e}^{-k_{g}}$ \\
Richards & $W_{r}^{-n}$ & $W_{r f}^{-n}$ & $W_{r 0}^{-n}-W_{r f}^{-n}$ & $\mathrm{e}^{-k_{r}}$ \\
\hline
\end{tabular}

The $y_{1}$ and $y_{2}$ parameters are the values of $y$ at $t_{1}$ and $t_{2}$, respectively. S-shaped curves are obtained for $v>0$ and $\mu>1$ (Keen, 1988). At given $\mu$, parameter $v$ determines the measure of curvation of the upper and lower curves. Parameter $\mu$ regulates the curvature of the upper curve in relation to the lower curve. The Schnute model encompasses eleven submodels, including the exponential $(v<0, \mu=1)$, monomolecu$\operatorname{lar}(v>0, \mu=1)$, logistic $(v>0, \mu=-1)$, Gompertz $(v>0, \mu=0)$, and Richards $(v>$ $0, \mu<0)$ growth models.

\section{References}

Anonymous, 1993. Genstat 5 release 3 reference manual. Clarendon Press, Oxford, 796 pp.

Charles-Edwards, D.A., D. Doley \& G.M. Rimmington, 1986. Modelling plant growth and development. Academic Press, Sydney, 235 pp.

France, J. \& J.H.M Thornley, 1984. Mathematical models in agriculture. Butterworths, London, 335 pp.

Hammond J.C. \& D. Kirkham, 1949. Growth curves of soybeans and corn. Agronomy Journal 41: 23-29.

Heinen, M., A. de Jager \& H. Niers, 1991. Uptake of nutrients by lettuce on NFT with controlled composition of the nutrient solution. Netherlands Journal of Agricultural Science 39: 197-212.

Hunt, R, 1978. Plant growth analysis. The Institute of Biology's Studies in Biology no 96. Edward Arnold, London, $67 \mathrm{pp}$.

Hunt, R, 1982. Plant growth curves. The functional approach to plant growth analysis. Edward Arnold, London, $248 \mathrm{pp}$.

Keen, A, 1988. FITG: A Genstat-procedure for fitting data to the general growth equation as described by Schnute (1981) (In Dutch). Report LWB-88-19, GLW-DLO, Wageningen, 42 pp.

Mitscherlich E.A., 1928. Die zweite Annäherung des Wirkungsgesetzes der Wachstumsfaktoren. Zeitschrift für Pflanzenernährung 12: 273-282.

Richards F.J., 1959. A flexible growth function for empirical use. Journal of Experimental Botany 10: 290-300.

Schnute, J, 1981. A versatile growth model with statistically stable parameters. Canadian Journal of Fisheries and Aquatic Science 38:1128-1140. 


\section{Appendix A}

The partial fractioning method is demonstrated by integrating the Richards growth equation Eq. (21) (leaving out the subscript $r$ for convenience)

$$
\frac{\mathrm{d} W}{\mathrm{~d} t}=k W \frac{W_{f}^{n}-W^{n}}{n W_{f}^{n}} .
$$

Integrating Eq. (A-1) over $t=0, W=W_{0}$ and $t=t, W=W$ yields

$$
\int_{w_{0}}^{w} \frac{n W_{f}^{n}}{W_{f}^{n}-W^{n}} \mathrm{~d} W=\int_{0}^{t} k \mathrm{~d} t=k t
$$

The left integral of Eq. (A-2) can be written as follows (partial fractioning)

$$
\int_{w_{0}}^{w} \frac{n W_{f}^{n}}{W_{f}^{n}-W^{n}} \mathrm{~d} W=n \int_{w_{0}}^{w}\left(\frac{A}{W}+\frac{B}{W_{f}^{n}-W^{n}}\right) \cdot \mathrm{d} W
$$

Values for $A$ and $B$ can be obtained from

$$
A\left(W_{f}^{n}-W^{n}\right)+B W=W_{f}^{n}
$$

yielding $A=1$ and $B=W^{n-1}$. The left integral of Eq. (A-2) can now be written as

$$
\int_{w_{0}}^{w} \frac{n W_{f}^{n}}{W_{f}^{n}-W^{n}} \mathrm{~d} W=n \int_{w_{0}}^{w}\left(\frac{1}{W}+\frac{W^{n-1}}{W_{f}^{n}-W^{n}}\right) \mathrm{d} W=n \cdot \int_{w_{0}}^{w} \frac{1}{W} \mathrm{~d} W+n \int_{w_{0}}^{w} \frac{W^{n-1}}{W_{f}^{n}-W^{n}} \mathrm{~d} W .
$$

With $\mathrm{d} W^{n}=n W^{n-1} \mathrm{~d} W$ the left integral of Eq. (A-2) now becomes

$$
\int_{w_{0}}^{w} \frac{n W_{f}^{n}}{W_{f}^{n}-W^{n}} \mathrm{~d} W=n \int_{w_{0}}^{w} \frac{1}{W} \mathrm{~d} W-\int_{w_{0}}^{w} \frac{1}{W^{n}-W_{f}^{n}} \mathrm{~d} W^{n} .
$$

The solution of the left integral of Eq. (A-2) follows easily from Eq. (A-6)

$$
\int_{W_{0}}^{w} \frac{n W_{f}^{n}}{W_{f}^{n}-W^{n}} \mathrm{~d} W=n \ln \frac{W}{W_{0}}-\ln \left(\frac{W^{n}-W_{f}^{n}}{W_{0}^{n}-W_{f}^{n}}\right)=\ln \left(\frac{W^{n} W_{0}^{n}-W_{f}^{n}}{W_{0}^{n} W^{n}-W_{f}^{n}}\right) .
$$

From Eq. (A-2) and Eq. (A-7) it follows that

$$
\frac{W^{n}}{W_{0}^{n}} \frac{W_{0}^{n}-W_{f}^{n}}{W^{n}-W_{f}^{n}}=\mathrm{e}^{k t}
$$

Equation (A-8) can be solved simply for $W$ yielding Eq. (23). 